\title{
Response to Pegylated Interferon in a COVID-19 Positive Male with Metastatic Jejunal Neuroendocrine Tumor Treated with Everolimus
}

\author{
Arthur Frankel ${ }^{1}$, Wai Yip ${ }^{1}$, Eknath Naik$^{1}$, and Hans Hasselbalch ${ }^{2}$ \\ ${ }^{1}$ West Palm Beach VA Medical Center \\ 2Zealand University Hospital
}

February 18, 2021

\begin{abstract}
A 61 years old male had minimally symptomatic SARS-CoV-2 infection while taking everolimus. He remained RT-PCR positive for viral RNA for 52 days. Pegylated interferon for 4 weeks led to viral RNA clearance. The observations support consideration and further evaluation of combination therapy with everolimus plus interferon for COVID-19.
\end{abstract}

Response to Pegylated Interferon in a COVID-19 Positive Male with Metastatic Jejunal Neuroendocrine Tumor Treated with Everolimus

Arthur E. Frankel, MD ${ }^{1 *}$; Wai C. Yip ${ }^{1}$; Eknath Naik, MD, PhD ${ }^{1}$; Hans C. Hasselbalch, MD, PhD ${ }^{2}$.

${ }^{1}$ Department of Medicine, West Palm Beach VA Medical Center, West Palm Beach, FL; ${ }^{2}$ Department of Hematology, Zealand University Hospital, Roskilde, Denmark.

*Correspondence to: Arthur E. Frankel, MD, Bldg 15 BA42, West Palm Beach VA Medical Center, West Palm Beach, FL 33410; telephone: 254-718-0781; email: afrankel59@gmail.com.

The study was conducted in accordance with the Declaration of Helsinki. Informed consent was obtained for the interferon therapy and case report.

Abstract word number 89; Text word count 3690; Tables 1.

Conflicts of interest: None

\section{Key Clinical Message}

A 61 years old male had minimally symptomatic SARS-CoV-2 infection while taking everolimus. He remained RT-PCR positive for viral RNA for 52 days. Pegylated interferon for 4 weeks led to viral RNA clearance. The observations support consideration and further evaluation of combination therapy with everolimus plus interferon for COVID-19.

\section{Introduction}

SARS-CoV-2 is a positive-sense, single stranded RNA in the Coronaviridae family of viruses ${ }^{1}$. While most cases of infection present with mild to moderate symptoms consisting of fever, fatigue, dry cough, headache, hypogeusia, anosmia, nausea and diarrhea ${ }^{2}$; roughly $15 \%$ of patients develop a severe disease phenotype requiring hospitalization, most commonly due to dyspnea and hypoxia ${ }^{3,4}$. Laboratory findings of severe infection include leukopenia, prolonged prothrombin time, and elevated serum concentrations of D-dimer, 
lactate dehydrogenase (LDH), ferritin, and c-reactive protein (CRP $)^{5}$. Chest computed tomography classically demonstrates bilateral ground glass opacities ${ }^{1}$. Severe pathology associated with SARS-CoV-2 infection involves a hyperactive immune response to the virus resulting in a sudden, acute increase in pro-inflammatory cytokines, termed "the cytokine storm" ${ }^{6}$. Key pro-inflammatory cytokines upregulated in this process include interleukin 6 (IL-6) and tumor necrosis factor-alpha $(\mathrm{TNF}-\alpha)^{6}$. Elevated cytokine levels prompt an influx of various immune cells into the site of infection, leading to tissue destruction, acute respiratory distress syndrome, septic shock and multiorgan failure ${ }^{7}$. Several mRNA and adenovirus vaccines targeting the spike protein have been approved ${ }^{8}$, but they appear to have reduced efficacy against the multiple higher infectivity and immune evading variants (e.g., spike protein mutations A222V, N501Y, or E484K/N501Y/K417N) that have arisen ${ }^{9,10}$. Thus, general SARS-CoV-2 therapies are needed. Early administration of monoclonal antibodies to the spike protein, RNA-dependent RNA polymerase inhibitor remdesivir and dexamethasone and late administration of enoxaparin may modify the natural history of the infection ${ }^{11-15}$, but additional treatments are needed. We previously reported the activity of a ruxolitinib plus interferon combination ${ }^{16}$. In this case report, we examine another combination that may show utility for SARS-CoV-2 infections.

Well differentiated small intestinal neuroendocrine tumors arise from serotonin-producing enterochromaffin cells and show epigenetic alterations frequently leading to PI3K/mTOR activation ${ }^{17}$. Upon metastases to liver and other sites, serotonin escapes liver metabolism, and the carcinoid syndrome occurs with flushing, diarrhea, and abdominal pain ${ }^{18}$. Treatments include sandostatin analogues, telotristat ethyl, ${ }^{177} \mathrm{Lu}$-DOTATATE, everolimus, sunitinib, interferon- $\alpha$, and surgical resection or ablation of metastases ${ }^{19}$. Metastatic small intestinal NET patients have a $70 \% 5$-year survival ${ }^{20}$.

PI3K/mTOR pathway inhibitors have been proposed as a therapy to target viral protein synthesis and the hyperinflammation associated with SARS-CoV- $2^{21}$. mTOR is the serine/threonine protein kinase catalytic subunit of the mTORC1 complex with LST8, PRA540 and raptor. Targets of mTOR include phosphorylation inactivation of $4 \mathrm{E}-\mathrm{BP}-1$ and $\mathrm{LARP}-1^{22,23}$. The result is release of eIF- $4 \mathrm{E}$ translation initiation factor and stimulation of cap-dependent, 5'-terminal oligopyrimidine tract viral RNA translation. SARS-CoV-2 proteins $\mathrm{N}$ and nsp1 assist in this seminal virus infectivity step ${ }^{24,25}$. Inhibitors of mTOR block viral RNA synthesis $^{23}$. SARS-CoV-2 hyper-inflammation is associated with the NLRP3 inflammasome containing the tripartite NLRP3 sensor protein, ASC adapter protein, and caspase- $1^{26}$. Infection produces a "priming" signal with viral RNA binding and signaling through Toll-like receptors or NOD-like receptors leading to $\mathrm{NF}-x \mathrm{~B}$ nuclear translocation, and NLRP3 and procaspase gene expression ${ }^{27}$. Then SARS-CoV-2 viroporins E, Orf3a and Orf8a cause cellular potassium efflux, NLRP3 oligomerization, ASC recruitment, and, finally, procaspase-1 recruitment and cleavage ${ }^{27}$. Orf8b aids the steps by binding the leucin-rich repeat domain of NLRP3 facilitating oligomerization. Gasdermin D is cleaved by caspase 1 and pyroptosis occurs along with inflammatory cytokine release. mTOR stimulates the NLRP3 inflammasome by increased mitochondrial reactive oxygen species formation and inhibiting autophagy ${ }^{28}$. As predicted, mTOR inhibitors inhibit NLRP3 inflammasome induced hyper-inflammation in vitro and in vivo ${ }^{29,30}$.

Interferons signal through cell surface interferon receptors to JAK and STAT proteins and ultimately induce interferon-stimulated genes (ISGs) that halt viral translation via protein kinase receptor (PKR) and block viral NLRP3-induced inflammation via STAT1 ${ }^{31,32}$. SARS-CoV-2 proteins block interferon activity via multiple pathways. SARS-CoV-2 induced inflammasome caspases degrade interferon and interferon signaling polypeptides $^{33}$. Over a dozen SARS CoV-2 proteins block early interferon induction or activity ${ }^{31}$. Late in the natural history, infection is associated with excessive inflammatory cytokinemia including interferons with dysfunctional $\mathrm{T}$ and NK cell responses ${ }^{34}$.

We present the case of a 61 -years-old male found to be SARS-CoV-2 positive who was relatively asymptomatic while taking everolimus for co-existing metastatic neuroendocrine tumor but displayed a prolonged period of nasal swab PCR positivity. Administration of pegylated interferon was followed by prompt clearance of viral RNA by PCR. We hypothesize that the combination of everolimus with interferon may be useful in the acute COVID-19 setting to induce viral clearance with reduced risk of cytokine storm. 


\section{Methods}

Informed consent to monitor, treat and report the subject was obtained from the patient and approved by the West Palm Beach VAMC Research \& Development Committee. Nasopharyngeal swab specimens were obtained from the patient. Samples were shipped to Orlando for performance of the Gene Xpert Cepheid Innovation XPRSARS-COv2-10 RT PCR assay measuring viral N2 sequence abundance. The limit of detection was 0.01 plaque forming units $/ \mathrm{mL}(\mathrm{CFU} / \mathrm{mL})$ or 250 viral RNA copies $/ \mathrm{mL}$ with first cycle number above background $(\mathrm{Cq})$ of 39 . Blood samples were collected using a serum separator tube and serum also shipped to Orlando for performance of the Abbott Architect anti-N SARS CoV-2 IgG assay with results calculated as chemiluminescence ratios of sample to control with negative ratios being $<1.4$.

\section{Results/Case Presentation}

A 61-year-old Caucasian male veteran developed diarrhea, flushing, pruritis, and abdominal pain in June, 2019. Computerized tomography scans showed a jejunal mass and liver lesions. Liver biopsy confirmed well differentiated neuroendocrine tumor. Blood counts and chemistries were normal, but serotonin was $1246 \mathrm{ng} / \mathrm{mL}$ (normal 50-200ng/mL), chromogranin A 781ng/L (normal <39ng/L) and 24 hour urine 5hydroxyindoleacetic acid was $91 \mathrm{mg}$ (normal $<6 \mathrm{mg}$ ). He was treated with lanreotide $120 \mathrm{mg}$ subcutaneously weekly and everolimus 10mg orally daily. He did well until June 27, 2020 when he noted mild lightheadedness, nausea, cough, headache, clear sputum production, hypogeusia, and anosmia and had a positive SARS-CoV-2 nasal swab RT-PCR test (Table 1). He remained at home, and his symptoms resolved within several days. However, his RT-PCR assay remained positive repeatedly for 52 days (Table 1). Because of hospital restrictions at the time on RT-PCR positive patients, he was unable to receive his monthly clinic lanreotide injections. His carcinoid symptoms recurred, and he required breakthrough octreitide acetate $200 \mathrm{mcg}$ subcutaneous injections every 8 hours at home. To facilitate viral RNA clearance, we elected to try pegylated interferon alpha-2a as treatment for both his neuroendocrine tumor and his COVID-19. After informed consent and approval by the West Palm Beach VA Medical Center Administration, Pharmacy and Research \& Education Committee, the patient received four weekly subcutaneous injections of 90 mcg pegylated interferon- $\alpha$-2a. His RT-PCR rapidly cleared within one week of treatment, and he was able to resume somatostatin analogue therapy at the oncology clinic. Initial anti-N SARS-CoV-2 IgG and IgM antibodies were absent from his blood (IgG 0.03 and IgG 0.02), but by January, 2021 he had measurable antibodies (IgG 2.07 and IgM 2.18). He remained asymptomatic and is undergoing additional treatments for his metastatic neuroendocrine tumor.

\section{Discussion}

SARS-CoV-2 is associated with a wide range of symptoms ranging from a mild clinical phenotype with fever and cough to severe respiratory and/or multi-organ failure. SARS-CoV-2 has considerable morbidity and mortality, particularly among older patients with co-morbidities ${ }^{35}$. A significant factor contributing to the morbidity and mortality of this infection is the pulmonary and systemic inflammatory response as noted above ${ }^{36}$. We were struck by the minimal clinical findings in this high-risk, male with a co-existing malignancy. Our patient was on chronic everolimus therapy for metastatic neuroendocrine tumor. As noted above, everolimus is an mTOR inhibitor that may target both viral replication and inflammation. We speculate whether our patient's minimal clinical symptoms throughout his infection could be linked to the anti-inflammatory effect of the drug. Everolimus may reduce the SARS-CoV-2 inflammatory state, improve the quality of life, and perhaps prolong survival from this devastating disease. This speculation is supported by several case studies. Among 18 hospitalized COVID-19 positive renal transplant patients, three were on chronic everolimus ${ }^{37}$. A 60 years old male and a 40 years old female were maintained on everolimus and did well; one 40 years old male had everolimus stopped and died from infection complications. Among 10 COVID-19 tuberous sclerosis/lymphangioleiomatosis patients, five were on sirolimus or everolimus and only one was briefly hospitalized and recovered; four of five patients not on mTOR inhibitors were hospitalized and one died $^{38}$. A 45 years old T3 paraplegic pancreas-kidney transplant male with asthma and chronic renal insufficiency maintained on everolimus developed RT-PCR positive SARS-CoV-2 and had an eight days hospitalization with transient symptoms ${ }^{39}$. Among 111 SARS-CoV-2 positive liver transplant 
patients, immunosuppression with mycophenolate was associated with severe disease (risk ratio $=5.3$ ); immunosuppression with mTOR inhibitors - tacrolimus or everolimus yielded fewer severe cases (tacrolimus risk ratio $=0.54$ and everolimus risk ratio $=0.77)^{40}$.

An interesting facet of this case is the sustained positivity of the patient's SARS-CoV-2 test. He was repeatedly tested for viral RNA clearance by nasal swab RT-PCR secondary to his immunocompromised state, and because he required a negative test prior to treatment at the oncology clinic. Many SARS-CoV-2 infected individuals have persistently positive RT-PCR tests for weeks to months after clinical recovery ${ }^{41}$. Based on viral culture, the percent of these individuals who remain infectious approaches zero by 10 to 15 days after the onset of symptoms ${ }^{41-43}$. However, shedding of infectious SARS-CoV-2 has been demonstrated by viral culture or inferred by the presence of subgenomic RNA in a subset of individuals, including immunosuppressed hosts, for months following infection ${ }^{44,45}$. We lack Cq values for multiple tests on our patient, but the reported positivity implies a Cq less than 39. Likely our patient had low quantities of viral RNA or viral RNA fragments that were non-infectious.

The persistent positivity of his SARS-CoV-2 testing may be potentially secondary to the immunosuppressive effects of everolimus ${ }^{46}$. mTOR inhibitors inhibit dendritic cell maturation and function and $\mathrm{T}$ cell proliferation. In our case, possible hampered anti-viral defense was reversed with interferon supplementation with subsequent $\mathrm{T}$ cell activation to fight the SARS-CoV-2 infection. Our patient was able to clear the viral RNA after the administration of the first of a total of four treatments of pegylated interferon- $\alpha 2 \mathrm{a}$ while continuing treatment with everolimus. Presence of RT-PCR positivity for one to two months after SARS-CoV-2 infection is not rare ${ }^{47}$, and does not always represent infectious virions.

Interferons have been successfully used in the treatment of viral infections, such as hepatitis $\mathrm{C}$, multiple sclerosis, hematologic malignancies, in particular the Philadelphia-negative myeloproliferative neoplasms and neuroendocrine tumors ${ }^{48-50}$. In SARS-CoV-2, interferon therapy in phase 2 and phase 3 randomized clinical trials have shown reduced the duration of virus infection, reduced inflammatory markers including IL6 and CRP and reduced mortality when administered early ${ }^{51-58}$. As a note of caution, type I interferons administered in later stages may cause progressive tissue damage leading to a deleterious hyperinflammation characterized by the excessive macrophage activation and hypercoagulation seen in patients with acute disease $^{36}$. Interestingly, pharmacologic interferon treatment inhibits inflammation early by repressing the NLRP3 inflammasome via STAT1 ${ }^{32}$. We hypothesized that administration of interferon in our patient who was minimally symptomatic would strengthen anti-viral defense and potentially lead to viral RNA clearance. Our results support the hypothesis.

\section{Conclusion}

The availability of vaccines will reduce the number of acute cases of COVID-19. However, acute cases will continue to exist, requiring therapeutic interventions to reduce toxicities and improve survival. Reducing the cytokine storm appears to be crucial in preventing end organ damage which is associated with high mortality ${ }^{59}$. Genetic and immunologic studies of hospitalized COVID-19 subjects showed mutations yielding increased TYK2 or decreased IFNAR2 expression or inactivating mutations in interferon pathway genesIRF3, IRF7, IFNAR1/2, TBK1 or TLR3 or autoantibodies to interferons had more severe disease ${ }^{60-62}$. These subjects suffered increased inflammatory cytokines and absent anti-viral interferons. Targeted immune regulation to reverse this state may provide substantial benefit in SARS-CoV-2 infection. Our case suggests that everolimus plus pegylated interferon is a potential regimen for SARS-CoV-2 patients. This treatment combination may benefit select patients if used early in the disease. Future studies are needed to elucidate the potential therapeutic benefits and side effects of this regimen.

\section{No Acknowledgements}

\section{Disclosure of Conflicts of Interest}

None of the authors has a relevant conflict of interest.

\section{Author Contributions}


Author 1: Conceived of study, obtained permissions, accessed agents, provided patient care, wrote draft manuscript.

Author 2: Assisted in patient care and obtained SARS-CoV-2 nasal swabs and reviewed manuscript.

Author 3: Assisted in collecting patient data and reviewed manuscript.

Author 4: Aided in planning patient treatment and in review of manuscript.

\section{References}

1. Dhama K, Khan S, Tiwari R, et al. Coronavirus disease 2019-COVID-19.Clinical Microbiol Rev . 2020;33(4):e00028-20.

2. Huang C, Wang Y, Li X, et al. Clinical features of patients infected with 2019 novel coronavirus in Wuhan, China. Lancet . 2020;395(10223):497-506.

3. Price-Haywood EG, Burton J, Fort D, Seoane L. Hospitalization and mortality among black patients and white patients with COVID-19. New Engl J Med . 2020;382(28):2634-2543.

4. Kashyap S, Gombar S, Yadlowsky S, et al. Measure what matters: counts of hospitalized patients are a better metric for health system capacity planning for a reopening. J Am Med Inform Assoc . 2020;27(7):10261131.

5. Liu Y, Yang Y, Zhang C. Clinical and biochemical indexes for 2019-nCoV infected patients linked to viral load and lung injury.Sci China Life Sci .2020;63:364-374.

6. Ragab D, Salah Eldin H, Taeimah M, Khattab R, Salem R. The COVID-19 Cytokine Storm; What We Know So Far. Front Immunol. 2020;11:1446.

7. Tabary M, Khanmohammadi S, Araghi F, et al. Pathologic features of COVID-19: a concise review. Pathol Research \& Practice .2020;216:153097.

8. Batty CJ, Heise MT, Bachelder EM, et al. Vaccine formulation and clinical development for the prevention of severe acute respiratory syndrome coronavirus 2 infection. Adv Drug Del Reviews . 2021;169:168-189.

9. Reardon S. The most worrisome mutations in five emerging coronavirus variants. Scientific American . 2021; 324: Epub Jan 29.

10. Callaway E, Mallopaty S. Novavax offer first evidence that COVID vaccines protect people against variants. Nature. 2021;589:.Epub Jan 29.

11. Chen P, Nirula A, Heller B, et al. SARS-CoV-2 neutralizing antibody LY-CoV555 in outpatients with Covid-19. New Engl J Med . 2021; 384(3):229-237.

12. Weinreich DM, Sivapalasingam S, Norton T, et al. REGN-CoV2, a neutralizing antibody cocktails in outpacd, tients with Covid-19. New Engl J Med . 2021; 384(3):238-251.

13. Spinner CD, Gottlieb RL, Criner GJ, et al. Effect of remdesivir versus standard care on clinical status at 11 days in patients with moderate COVID-19. JAMA. 2020; 324(11):1048-1057.

14. Tomazini BM, Maia IS, Cavalcanti AB, et al. Effect of dexamethasone on days alive and ventilator-free in patients with moderate or severe acute respiratory distress syndrome and COVID-19. JAMA . 2020; 324(13):1307-1316.

15. Lemos ACB, do Espirito Santo DA, Salvetti MC, et al. Therapeutic versus prophylactic anticoagluation for severe COVID-19. Thromb Res . 2020; 196: 359-366.

16. Frankel AE,Reddy R, DeSouza KR, et al. Response to pegylated interferon in a COVID-19 positive elderly woman with primary myelofibrosis treated with ruxolitinib. Clinical Case Reports . 2021; in press. 
17. Asa SL, La Rosa S, Basturk O, et al. Molecular pathology of well-differentiated gastro-entero-pancreatic neuroendocrine tumors.Endocrine Pathol. 2021; Epub, Jan 18.

18. Gade AK, Olariu E, Douthit NT. Carcinoid syndrome. Cureus.2020; 12(3):e7186.

19. Oleinikov K, Avriel-Polak S, Gross DJ, et al. Carcinoid syndrome: updates and review of current therapy. Curr Treat Options Oncol . 2019;20(9):70.

20. Strosberg JR, Weber JM, Feldman M, et al. Prognostic validity of the American Joint Committee on Cancer Staging Classification for midgut neuroendocrine tumors. J Clin Oncol . 2013;31(4):420-425.

21. Husain A, Byrareddy SN. Rapamycin as a potential repurposed drug candidate for the treatment of COVID-19. Chem Biol Interact . 2020;331:109282.

22. Philippe L, van den Elzen AMG, Watson MJ, et al. Global analysis of LARP1 translation targets reveal tunable and dynamic features of 5'-TOP motifs. Proc Natl Acad Sci USA .2020; 117(10): 5319-5328.

23. Appelberg S, Gupta S, Akusjarvi SS, et al. Dysregulation of Akt/mTOR/HIF-1 signaling identified by proteo-transcriptomics of SARS-CoV-2 infected cells. Emerg Microbes Infect . 2020;9(1):1748-1760.

24. Schmidt N, Lareau CA, Keshishan H, et al. The SARS-CoV-2 RNA protein interactome in infected human cells. Nature Microbiol .2020;Epub Dec21.

25. Rao S, Hoskins I, Garcia PD, et al. Genes with 5'-terminal oligopyrimidine tracts preferentially escape global suppression of translation by the SARS-CoV-2 Nsp1 protein. bioRxiv .2020;Epub, Dec 18.

26. Toldo S, Bussani R, Nuzzi V, et al. Inflammasome formation in the lungs of patients with fatal COVID19. Inflammation Res.2021;70(1):7-10.

27. Freeman TL, Swartz TH. Targeting the NLRP3 inflammasome in severe COVID-19. Front Immunol $.2020 ; 11: 1518$.

28. Wang H, Zhang P, Sun L. Exogeneous hydrogen sulfide inhibits NLRP3 inflammasome mediated inflammation through promoting autophagy via the AMPK-mTOR pathway. Biol Open. 2019;8:bio043653.

29. Yang T, Feng X, Zhao Y, et al. Dexmedotamidine enhances autophagy via alpha2-AR/AMPK/mTOR pathway to inhibit the activation of NLRP3 inflammasome and subsequently alleviate liposaccharide induced acute kidney injury. Front Pharm. 2020;11:790.

30. Li X, Zhang X, Pan Y, et al. mTOR regulates NLRP3 inflammasome activation via reactive oxygen species in murine lupus. Acta Biochim Biophys Sin .2018;50(9):888-896.

31. Sa Ribero M, Jouvenet N, Dreux M, et al. Interplay between SARS-CoV-2 and the type I interferon response. PLoS Pathogens .2020;16(7):e1008737.

32. Guarda G, Braun M, Staehli F, et al. Type I interferon inhibits interleukin-1 production and inflammasome activation.Immunity .2011;34(2):213-223.

33. Ning X, Wang Y, Jing M, et al. Apoptotic caspases suppress type I interferon production via the cleavage of cGAS, MAVS, and IRF3.Molecular Cell . 2019;74:19-31

34. Jamilloux Y, Henry T, Belot A, et al. Should we stimulate or suppress immune responses in COVID-19? Cytokine and anti-cytokine interventions. Autoimmunity reviews. 2020;19(7):102567.

35. Li X, Xu S, Yu M, et al. Risk factors for severity and mortality in adult COVID-19 inpatients in Wuhan. J Allergy Clin Immunol.2020;146(1):110-118.

36. Merad M, Martin JC. Pathological inflammation in patients with COVID-19: a key role for monocytes and macrophages. Nat Rev Immunol. 2020;20(6):355-362. 
37. Devresse A, Belkhir L, Vo B, et al. COVID-19 infection in kidney transplant recipients. Kidney Medicine. 2020;2(4):459-466.

38. Peron A, La Briola F, Bruschi F, et al. Tuberous sclerosis complex (TSC), lymphangioleiomyo-matosis, and COVID-19. Am J Med Genet A . 2020;182(11):2479-2485.

39. Heron VC, Bach CAT, Holmes NE, et al. Complete recovery from COVID-19 in a kidney-pancreas transplant recipient: potential benefit of everolimus? BMJ Case Rep . 2021;14(1):e238413.

40. Colmenero J, Rodriguez-Peralvarez M, Salcedo M, et al. Epidemiological pattern, inicidence, and outcomes of COVID-19 in liver transplant patients. J Hepatol .2021;74(1):148-155.

41. Cevik M, Tate M, Lloyd O, et al. SARS-CoV-2, SARS-CoV, and MERS-CoV viral load dynamics, duration of viral shedding and inectiousness: a systematic review and meta-analysis. Lancet Microbe. 2020; Epub Nov 19.

42. Rhee C, Kanjilal S, Baker M, Klompas M. Duration of SARS-CoV-2 infectivity: when is it safe to discontinue isolation? Clin Infect Dis . 2020;Epub Aug 25.

43. Bullard J, Dust K, Funk D, et al. Predicting infectious SARS-CoV-2 from diagnostic samples. Clin Infect Dis . 2020;Epub May 22.

44. Avanzato VA, Matson MJ, Seifert SN, et al. Case study: prolonged infectious SARS-CoV-2 shedding from an asymptomatic immunocompromised individual with cancer. Cell . 2020;Epub Nov 4.

45. Rodriguez-Grande C, Adan-Jimenez J, Catalan P, et al. Inference of active viral replication in cases with sustained positive RT-PCRs from SARS-CoV-2. J Clin Microbiol . 2020;Epub Nov 25.

46. Thomson AW, Turnquist HR, Raimondi G. Immunoregulatory functions of mTOR inhibition. Nat Rev Immunol .2009;9(5):324-337.

47. Kim MC, Cui C, Shin KR, et al. Duration of culturable SARS-CoV-2 in hospitalized patients with Covid-19.New Engl J Med . 2021;384:Epub, Jan 27.

48. Bohn JP, Gastl G, Steurer M. Long term treatment of hairy cell leukemia with interferon-alpha: still a viable therapeutic option. Memo . 2016;9:63-65.

49. Hasselbalch HC, Holmstrom MO. Perspectives on interferon-alpha in the treatment of polycythemia vera and related myeloprolifeative neoplasms: minimal residual disease and cure? Semin Immunopath . 2019;41(1):5-19.

50. Kanderli RM, Spanjol M, Kollar A, et al. Therapeutic options for neuroendocrine tumors. JAMA Oncol $.2019 ; 5(4): 480-489$.

51. Fung SY, Yuen KS, Ye ZW, Chan CP, Jin DY. A tug-of-war between severe acute respiratory syndrome coronavirus 2 and host antiviral defence: lessons from other pathogenic viruses. Emerging microbes $\mathscr{B}$ infections. 2020;9(1):558-570.

52. Wang N, Zhan Y, Zhu L, e al. Retrospective multicenter cohort study shows early interferon therapy is associated with favora ble clinical responses in COVID-19 patients. Cell Host Microbe.2020;28(3):456-464.

53. Zhou Q, Chen V, Shannon CP, et al. Interferon-alpha2b treatment for COVID-19. Front Immunol. 2020;11:1061.

54. Hung IFH, Lung KC, Tso EYK, et al. Triple combination of interferon beta-1b, lopinavir-ritonavir, and ribavirin in the treatment of patients admitted to hospital with COVID-19: an open-label, randomised, phase 2 trial. Lancet . 2020;395(10238): 1695-1704.

55. Zheng F, Zhou Y, Zhou Z, et al. SARS-CoV-2 clearance in COVID-19 patients with Novaferon treatment: a randomized, open-label, parallel-group trial. Int J Infect Dis . 2020;99:84-91. 
56. Davoudi-Monfared E, Rahmani H, Hhalill H, et al. A randomized clinical trial of the efficacy and safety of interferon beta-1a in treatment of severe COVID-19. Antimicrob Agents Chemother.2020;64(9):e01061.

57. Pereda R, Gonzalez D, Rivero HB, et al. Therapeutic effetiveness of interferon-alpha2b against COVID19: the Cuban experience. J Interferon Cytokine Res . 2020;40(9):438-442.

58. Fu W, Liu Y, Liu L, et al. An open-label, randomized trial of the combination of IFN-kappa plus TFF2 with standard care in the treatment of patients with moderate COVID-19. EClinicalMedicine . 2020; $27: 100547$.

59. Ye Q, Wang B, Mao J. The pathogenesis and treatment of the 'Cytokine Storm' in COVID-19. J Infect. 2020;80(6):607-613.

60. Pairo-Castineira E, Clohisey S, Klaric L, et al. Genetic mechanism of critical illness in Covid-19. Nature . 2020; Epub Dec 11.

61. Zheng Q, Bastard P, Liu Z, et al. Inborn errors of type I IFN immunity in patients with life-threatening COVID-19. Science . 2020;370(6515):eabd4570.

62. Bastard P, Rosen LB, Zheng Q, et al. Autoantibodies against type I IFN in patients with life-threatening COVID-19. Science . 2020;370(6515):eabd4585.

Table 1. RT-PCR Cq values *

\section{Day}

$-17$

1

15

25

38

52

69

97

180

191

RT-PCR Cq, reverse transcriptase polymerase chain reaction cycle at which fluorescence detected above baseline. The value 